\title{
The molecular mechanisms underlying reduced E-cadherin expression in invasive ductal carcinoma of the breast: high throughput analysis of large cohorts
}

\author{
Mansour Alsaleem ${ }^{1,2} \cdot$ Michael S. Toss $\mathbb{1}^{1}$ - Chitra Joseph $\mathbb{1}^{1} \cdot$ Mohammed Aleskandarany ${ }^{1,3} \cdot$ Sasagu Kurozumi ${ }^{1}$. \\ Ibrahim Alshankyty ${ }^{4}$ Angela Ogden ${ }^{5}$ Padmashree C. G. Rida ${ }^{5} \cdot \operatorname{lan}$ O. Ellis ${ }^{1} \cdot$ Ritu Aneja ${ }^{5}$ Andrew R. Green ${ }^{1}{ }^{1}$ • \\ Nigel P. Mongan $\mathbb{1}^{6,7} \cdot$ Emad A. Rakha ${ }^{1,3}$
}

Received: 8 November 2018 / Revised: 23 December 2018 / Accepted: 28 December 2018 / Published online: 13 February 2019

(c) United States \& Canadian Academy of Pathology 2019

\begin{abstract}
E-cadherin is a tumor suppressor gene in invasive lobular breast cancer. However, a proportion of high-grade ductal carcinoma shows reduced/loss of E-cadherin. In this study, we assessed the underlying mechanisms and molecular implications of E-cadherin loss in invasive ductal carcinoma. This study used large, well-characterized cohorts of early-stage breast cancer-evaluated E-cadherin expression via various platforms including immunohistochemistry, microarray analysis using Illumina HT-12 v3, copy number analysis using Affymetrix SNP 6.0 arrays, and next-generation sequencing for differential gene expression. Our results showed $27 \%$ of high-grade invasive ductal carcinoma showed reduced/loss of E-cadherin membranous expression. $C D H 1$ copy number loss was in $21 \%$ of invasive ductal carcinoma, which also showed low $C D H 1$ mRNA expression $(p=0.003)$. $C D H 1$ copy number was associated with copy number loss of TP53, ATM, $B R C A 1$, and $B R C A 2(p<0.001)$. Seventy-nine percent of invasive ductal carcinoma with reduced $C D H 1$ mRNA expression showed elevated expression of E-cadherin transcription suppressors TWIST2, ZEB2, NFKB1, LLGL2, CTNNB1 $(p<0.01)$. Reduced/loss E-cadherin expression was associated with differential expression of 2143 genes including those regulating Wnt (FZD2, GNG5, HLTF, WNT2, and CER1) and PIK3-AKT (FGFR2, GNF5, GNGT1, IFNA17, and IGF1) signaling pathways. Interestingly, key genes differentially expressed between invasive lobular carcinoma and invasive ductal tumors did not show association with E-cadherin loss in invasive ductal carcinoma. We conclude that E-cadherin loss in invasive ductal carcinoma is likely a consequence of genomic instability occurring during carcinogenesis. Potential novel regulators controlling E-cadherin expression in invasive ductal carcinoma warrant further investigation.
\end{abstract}

\section{Introduction}

Worldwide, breast cancer accounts for $23 \%$ of total diagnosed cancer cases and is the second leading cause of cancer-related death among women [1]. In solid tumors, cell-cell decohesion is a recognized phenomenon allowing tumor cells to grow invasively into surrounding tissues [2]. E-cadherin, a calcium-dependent adhesion molecule encoded by the $\mathrm{CDH} 1$ gene located on chromosome 16q22.1 [3],

Supplementary information The online version of this article (https:// doi.org/10.1038/s41379-019-0209-9) contains supplementary material, which is available to authorized users.

Emad A. Rakha

Emad.Rakha@nottingham.ac.uk

Extended author information available on the last page of the article has an important role in gland formation, cell differentiation, polarity, and maintaining the integrity of epithelial cells [4]. Subsequently, decreased expression of E-cadherin, which is frequently seen in breast cancer, may lead to cellular de-differentiation and invasiveness [5, 6].

Reduced/loss of E-cadherin expression in the vast majority of invasive lobular carcinomas and lobular carcinoma in situ, together with loss of CDH1 gene copy number [7-9] or CDH1 gene mutation [10] in a large proportion of cases, suggests a plausible role for E-cadherin as a tumor suppressor gene [11,9]. However, there is limited evidence to support a role for E-cadherin as a tumor suppressor gene in invasive ductal carcinoma [12]. In fact, ductal carcinoma in situ and low-grade invasive ductal carcinoma generally show stronger E-cadherin membrane staining than that seen in the normal breast epithelial cells, denoting increased expression rather than a loss of 
expression [13]. Although some studies indicated that a proportion of invasive ductal carcinoma shows loss/ reduced E-cadherin protein expression, these tumors were typically high-grade aggressive tumors. Of note, accumulating evidence suggests that high-grade invasive ductal carcinoma are characterized by genomic instability with loss of increasing number of tumor suppressor genes during the carcinogenesis process that contributes to their aggressive behavior [12]. In addition, reduced/loss of E-cadherin expression is frequently associated with loss of estrogen expression, larger tumor size, and with the development of metastasis and recurrence [14-17]. These findings suggest that E-cadherin loss occurs as a late event in the process of carcinogenesis arising in association with or as a part of genomic instability rather than as an early neoplastic event as seen in invasive lobular carcinoma [13, 18, 19]. However, the reasons for dysregulation of E-cadherin protein expression remain ill-defined [20].

We therefore aimed to study the mechanisms of reduced/ loss E-cadherin expression in high-grade invasive ductal carcinoma compared with invasive lobular carcinoma and its potential molecular implications.

\section{Materials and methods}

\section{Study cohort}

This study was conducted on multiple well-characterized cohorts of high-grade invasive ductal carcinoma using different molecular techniques (Supplementary Table 1). First, a well-characterized cohort of primary grade 3 invasive ductal carcinoma from patients presenting to Nottingham City Hospital between 1989 and $1998(n=813)$, and for whom detailed clinicopathologic data were available was used to determine E-cadherin expression using immunohistochemistry [12]. The mean patient age was 52 years (range 18-71) and tumor size ranged in diameter from 0.1 to $5 \mathrm{~cm}$ at time of presentation, with a mean tumor size of $2 \mathrm{~cm}$ (Supplementary Table 2). To understand the molecular biology of E-cadherin expression, high-grade invasive ductal carcinoma $(n=883)$ cases in the Molecular Taxonomy of Breast Cancer International Consortium (METABRIC) cohort [21] were used to investigate copy number alterations and $C D H 1$ mRNA expression. The mean patient age was 59 years (range 26-96) and mean tumor size at time of presentation was $3 \mathrm{~cm}$ (range from 1 to $18 \mathrm{~cm}$ ). In the METABRIC-invasive ductal carcinoma series, DNA/RNA was isolated from fresh frozen samples and transcriptional profiling was obtained using the Affymetrix SNP 6.0 Illumina Total Prep RNA Amplification Kit and Illumina Human HT-12 v3 Expression Bead Chips (Ambion, Warrington, UK). Copy number alteration was considered at the gene level by segments and the Šidák correction [22], whereas gene expression data were pre-processed and normalized as described previously [21]. In this cohort, patients with estrogen-positive tumor and /or lymph node negative at time of diagnosis did not receive adjuvant chemotherapy, whereas those with estrogen-negative tumors and lymph node-positive status received adjuvant treatment. Next-generation RNA sequencing (RNA-Seq) was conducted on an additional triple negative breast cancer cohort $(n=106)$ to investigate E-cadherin reduced/loss expression in this subtype of breast cancer. The mean patient age was 48 (range 27-69) and tumors size ranged in diameter from 1 to $6 \mathrm{~cm}$ at time of presentation, with a mean tumor size of $2 \mathrm{~cm}$ (Supplementary Table 2).

\section{Immunohistochemistry staining and scoring}

Mouse monoclonal anti-E-cadherin antibody [Cl;4A2C7, Ref\#180223, LOT 954621A, Invitrogen, UK] was used to assess protein expression on immunohistochemically stained tissue sections after prior validation of the antibody by western blotting using MDA-MB-231 and MDA-MB157 breast cancer cell lysates (obtained from American Type Culture Collection, Rockville, MD, USA). Immunohistochemistry staining procedure was performed using Novocastra Novolink TM Polymer Detection Systems kit (Code: RE7280-K, Leica, Biosystems, UK) on $4 \mu \mathrm{m}$ tissue microarray sections [20]. Sections were incubated for $24 \mathrm{~h}$ with the anti-CDH1 antibody diluted to a concentration of 1:25. Scoring of membranous protein expression was performed using the modified histo-score [23]. We used the lower quartile from the modified histochemical score value (i.e., 85) to stratify the cohort into high and reduced/loss E-cadherin expression groups. Cases in the METABRIC cohort were stratified using a similar approach for total CDH1 mRNA expression. Copy number alteration and CDH1 mRNA expression were correlated with E-cadherin protein expression in the same cases where available $(n=131)$.

\section{RNA sequencing}

RNA-Seq was performed on representative formalinfixed paraffin-embedded blocks of triple negative breast cancers $(n=106)$, which had also been assessed histopathologically for tumor burden. Invasive tumor cells were micro-dissected from unstained tissue sections where tissue burden was at least $50 \%$ of the tissue section area. Micro-dissected tissues were deparaffinized, rehydrated, and centrifuged to remove excess ethanol. RNA was extracted using the Omega Mag-Bind XP formalin-fixed paraffin-embedded RNA isolation kit (Omega, M2595-01) 
and Kingfisher Flex magnetic particle separator (ThermoFisher) as per the manufacturer's instructions. RNA was measured with a Nanodrop 2000c spectrophotometer (Thermo Scientific). First-strand cDNA synthesis was performed on $\sim 100 \mathrm{ng}$ RNA at $25^{\circ} \mathrm{C}$ for $10 \mathrm{~min}, 42{ }^{\circ} \mathrm{C}$ for $15 \mathrm{~min}$, and $70^{\circ} \mathrm{C}$ for $15 \mathrm{~min}$ using random hexamers and ProtoScript II Reverse Transcriptase (New England BioLabs, Ipswich, MA). Second-strand synthesis and RNA-Seq libraries were prepared using the Illumina TruSeq RNA access library kit (Illumina, RS-301-2002) and sequenced on an Illumina HiSeq 2500 using PE75 run chemistry. The targeted read count was $60 \mathrm{M}$ total reads per sample. Sequencing was performed at the Emory Integrated Genomics Core Facility, Emory University, Atlanta, USA. Raw FastQ sequence reads files were quality assessed and adapter processed using the trim galore wrapper for Fastqc and Cutadapt with reads with phred scores $>30$ retained. The resultant quality-trimmed reads were aligned to the hg38 (GRCh38.83) build of the human genome using the STAR aligner. Transcript abundance quantification were performed using HTSEQ [34]. Only one sample per patient was included in downstream analyses by random selection. Differential gene expression was assessed using Robina implementation of Edge-R [24].

\section{Pathway analysis}

The online public available web-based gene set analysis tool, Webgestalt, (http://www.webgestalt.org/option.php) was used to identify differentially regulated canonical pathways. This pathway analysis was based on transcripts differentially expressed at the $p<0.05$ level and generated by Robina analysis, including only unbiased hits with significant $z$-scores based on network-adjusted $p$-values $<0.05$ using KEGG pathway database [25].

\section{Statistical analysis}

IBM SPSS 24.0 (Chicago, IL, USA) software was used for statistical analysis. The $\chi^{2}$-test was used to assess the effect of copy number alteration on reduced/loss of $\mathrm{CDH} 1 \mathrm{mRNA}$ expression. Furthermore, we evaluated copy number alteration of established tumor suppressor genes in cases that exhibited reduced/loss of $C D H 1$ mRNA expression and copy number loss via copy number alteration in the METABRIC cohort, to infer genetic instability as the likely driver of the reduced/loss of $\mathrm{CDHI}$ mRNA expression using $\chi^{2}$-test. Mann-Whitney test was used to compare the expression of CDHI mRNA expression with expression of well-established transcription factors affecting E-cadherin expression [26]. Furthermore, we evaluated expression of a set of genes previously demonstrated to have $93 \%$ predictive accuracy in distinguishing invasive lobular carcinoma from invasive ductal carcinoma via the prediction analysis for microarrays test [27]. Expression of proteins related to DNA repair and proliferation were compared with expression of the E-cadherin protein using the Mann-Whitney test. Furthermore, the association of E-cadherin protein expression with that of transcription factors mRNA expression (assessed using next-generation sequencing-HTSEQ values) was evaluated using the Mann-Whitney test. Two-tailed $p$-value $<0.05$ was considered as statistically significant. RNA-Seq values were expressed as SEMs in GRAPH PAD PRISM v.7 for data presentation.

\section{Results}

\section{Evaluation of E-cadherin protein expression in the high-grade invasive ductal breast carcinoma cohort $(n=813)$}

The specificity of E-cadherin antibody was validated by western blotting that showed a single specific band at the expected molecular weight ( $100 \mathrm{kDa})$. A total of 217/813 (27\%) of high-grade invasive ductal carcinoma and 46/106 (43\%) of triple negative breast cancer showed reduced/loss membrane expression of E-cadherin. Within the METABRIC cohort, reduced/loss CDH1 mRNA expression was observed in 208/883 (23\%) cases. Furthermore, triple negative breast cancer showed reduced/loss of $\mathrm{CDHI}$ mRNA expression in 90/235 (38\%) cases. Reduced/loss CDHI mRNA expression cases were observed in 104 cases of the basal (37\%), 18 cases of the HER2 enriched (11\%), 40 cases of luminal A (27\%), 29 cases of luminal B (12\%), and 17 cases of the Normal-like (29\%) molecular subtypes (Supplementary Table 3 and Supplementary Figure 1). In the subset of cases that were included in the METABRIC dataset $(n=131)$, there was a positive linear correlation between $C D H I$ mRNA and the dichotomized E-cadherin protein expression $(r=0.27, p=0.002)$.

Reduced/loss E-cadherin protein expression was associated with GammaH2AX $(p<0.0001)$ and phosphatase and tensin homolog (PTEN) $(p=0.003)$ protein expression (Table 1).

\section{E-cadherin copy number alteration in ductal breast cancer}

To investigate whether reduced/loss of E-cadherin expression in the invasive ductal carcinoma cases is due to copy number alteration, we examined copy number alteration and $C D H 1$ mRNA levels. We observed that 44/208 $(21 \%)$ of cases showed significant association between loss of CDH1 copy number and reduced/loss $\mathrm{CDH} 1$ 
Table 1 Correlation between level of proteins associated with altered E-cadherin expression in high-grade invasive breast cancer cohort $(n=813)$

\begin{tabular}{|c|c|c|c|c|c|c|c|c|}
\hline \multirow[t]{2}{*}{ Identifier } & & \multirow[t]{2}{*}{$\begin{array}{l}\text { Protein of } \\
\text { interest }\end{array}$} & \multicolumn{2}{|c|}{$\begin{array}{l}\text { E-cadherin } \\
\text { expression } \\
\text { frequency }\end{array}$} & \multicolumn{2}{|c|}{$\begin{array}{l}\text { Mean } \\
\text { RANK }\end{array}$} & \multirow[t]{2}{*}{$Z$ - score } & \multirow[t]{2}{*}{$p$-Value } \\
\hline & & & Low $(\%)$ & High $(\%)$ & Low & High & & \\
\hline \multirow[t]{11}{*}{ Protein level } & Transcription factor & TGFBeta1 & $143(27)$ & $371(73)$ & 257 & 258 & -0.04 & 0.96 \\
\hline & & TWIST2 & $130(25)$ & $376(75)$ & 259 & 251 & -0.60 & 0.540 \\
\hline & & ZEB & $126(26)$ & $352(74)$ & 240 & 239 & -0.19 & 0.840 \\
\hline & DNA repair family & BRCA1 & $178(26)$ & $491(74)$ & 336 & 335 & -0.05 & 0.950 \\
\hline & & BRCA2 & $132(26)$ & $375(74)$ & 248 & 256 & -1.19 & 0.230 \\
\hline & & GammaH2ax & $120(23)$ & 387 (77) & 221 & 264 & -3.48 & $<0.001$ \\
\hline & & RAD51 & $110(25)$ & $320(75)$ & 199 & 221 & -1.96 & 0.050 \\
\hline & $\begin{array}{l}\text { Proliferation and } \\
\text { other markers family }\end{array}$ & Ki67 & $174(25)$ & $501(75)$ & 329 & 341 & -1.14 & 0.250 \\
\hline & & PTEN & $104(28)$ & $260(72)$ & 164 & 189 & -2.93 & 0.003 \\
\hline & & ATM & $143(28)$ & $358(72)$ & 245 & 253 & -0.70 & 0.480 \\
\hline & & TP53 & $214(26)$ & $590(74)$ & 408 & 400 & -0.47 & 0.630 \\
\hline
\end{tabular}

Significant $p$-values are in bold
mRNA expression $(p=0.003)$ (Supplementary Table 4). Only one case with copy number loss did not show any association with the transcription factors investigated, while the remaining cases showed upregulation of one or more transcription factors (Supplementary Table 5). Interestingly, $77 \%$ of tumors presenting with reduced/ loss $C D H 1$ mRNA expression did not show $C D H 1$ copy number loss, indicating that other mechanisms are implicated. Subsequently, investigating the triple negative tumors, only $7 / 90$ (8\%) of cases showed copy number to be associated with reduced/loss $C D H 1$ mRNA expression. However, there was no statistical association between copy number loss and reduced/loss of $C D H 1$ mRNA expression $(p=0.10)$ (Supplementary Table 6). More importantly, among those cases, only 1 (copy number loss) case did not show any association with any transcription factors, while the rest of the 6/90 (7\%) cases (copy number loss) showed upregulation of one or more transcription factors (Supplementary Table 7). Moreover, 83/90 (92\%) of triple negative tumors with reduced/loss $C D H 1$ mRNA expression showed neutral/amplified $C D H 1$ copy number expression.

In addition, reduced/loss $C D H 1 \mathrm{mRNA}$ expression in invasive ductal carcinoma showed copy number loss of multiple well-established breast cancer tumor suppressor genes located at different chromosome loci: TP53, ATM, $B R C A 1$, and BRCA2 $(p<0.001)$ (Supplementary Table 8).

\section{Expression of E-cadherin suppressor transcription factors}

In cases with reduced/loss E-cadherin expression $(n=208)$ from the METABRIC cohort, upregulated
mRNA expression was observed with ZEB2 (56\%), TWIST2 (54\%), NFKB1 (54\%), ZEB1 (53\%), TWIST1 (52\%), SLUG (51\%), SNAIL (50\%), GSK3BETA (49\%), TGFB1 (47\%), LLGL2 (38\%), and CRUMBS3 (34\%). Only $4 \%$ of the cases were affected by nine or more upregulated transcription factors (Supplementary Table 9 and Supplementary Figure 2). Upregulated expression of TWIST2, ZEB2, NFKB1, LLGL2, and CRUMBS3 were significantly associated with reduced/loss of $\mathrm{CDH} 1$ mRNA expression (Table 2). In triple negative breast cancer with reduced/loss E-cadherin expression, upregulated mRNA expression was observed with ZEB2 (63\%), SLUG (62\%), TWIST2 (59\%), TWIST1 (57\%), ZEBI (54\%), SNAIL (52\%), TGFB1 (51\%), GSK3BETA (50\%), NFKB1 (46\%), LLGL2 (24\%), and CRUMBS3 (24\%) (Supplementary Table 10 and Supplementary Figure 3). Only $3 \%$ of the cases harbored nine or more upregulated transcription factors (Supplementary Table 7). Upregulated expression of TWIST2, TWIST1, ZEB2, ZEB1, $S L U G, L L G L 2$, and CRUMBS3 were significantly associated with reduced/loss of $C D H 1$ mRNA expression (Table 3).

\section{Proteins associated with E-cadherin expression in invasive triple-negative ductal breast carcinoma}

There was no significant statistical correlation between reduced/loss of E-cadherin expression with transcription factors, DNA repair family, nor other markers such as ki67, ATM, and PTEN on the protein level in triplenegative breast cancer (Table $4 \mathrm{a}, \mathrm{b}$ and Supplementary Figure 4A) 
Table 2 Correlation between mRNA levels of the genes associated with altered Ecadherin expression in breast cancer in the METABRIC cohort

\begin{tabular}{|c|c|c|c|c|c|c|c|}
\hline \multirow[t]{2}{*}{ Identifier } & \multirow[t]{2}{*}{$\begin{array}{l}\text { Gene of } \\
\text { interest }\end{array}$} & \multicolumn{2}{|c|}{$\begin{array}{l}\text { E-cadherin } \\
\text { expression frequency }\end{array}$} & \multicolumn{2}{|c|}{$\begin{array}{l}\text { Mean } \\
\text { RANK }\end{array}$} & \multirow[t]{2}{*}{$Z$ - score } & \multirow[t]{2}{*}{$p$-Value } \\
\hline & & Low $(\%)$ & High (\%) & Low & High & & \\
\hline \multirow[t]{11}{*}{ Transcription factor mRNA level } & $T G F B 1$ & \multirow{11}{*}{$208(23)$} & \multirow{11}{*}{$675(77)$} & 443 & 442 & -0.07 & 0.940 \\
\hline & TWIST2 & & & 481 & 430 & -2.51 & 0.012 \\
\hline & TWIST1 & & & 466 & 434 & -1.58 & 0.110 \\
\hline & ZEB2 & & & 489 & 427 & -3.05 & 0.002 \\
\hline & ZEB1 & & & 445 & 441 & -0.21 & 0.830 \\
\hline & $S L U G$ & & & 463 & 436 & -1.33 & 0.180 \\
\hline & SNAIL & & & 457 & 437 & -0.94 & 0.340 \\
\hline & $N F K B 1$ & & & 489 & 427 & -3.06 & 0.002 \\
\hline & $L L G L 2$ & & & 349 & 470 & -6.00 & $<0.001$ \\
\hline & $G S K 3 B$ & & & 439 & 443 & -0.150 & 0.880 \\
\hline & CRUMBS & & & 335 & 475 & -6.93 & $<0.001$ \\
\hline
\end{tabular}

Significant $p$-values are in bold

\begin{tabular}{|c|c|c|c|c|c|c|c|}
\hline \multirow[t]{2}{*}{ Identifier } & \multirow[t]{2}{*}{$\begin{array}{l}\text { Gene of } \\
\text { interest }\end{array}$} & \multicolumn{2}{|c|}{$\begin{array}{l}\text { E-cadherin expression } \\
\text { frequency }\end{array}$} & \multicolumn{2}{|c|}{$\begin{array}{l}\text { Mean } \\
\text { RANK }\end{array}$} & \multirow[t]{2}{*}{ Z- score } & \multirow[t]{2}{*}{$p$-Value } \\
\hline & & Low $(\%)$ & $\mathrm{H}$ high $(\%)$ & Low & High & & \\
\hline \multirow[t]{11}{*}{ Transcription factor mRNA level } & $T G F B 1$ & \multirow{11}{*}{$90(38)$} & \multirow{11}{*}{$145(62)$} & 114 & 120 & -0.74 & 0.460 \\
\hline & TWIST2 & & & 130 & 110 & -2.16 & 0.030 \\
\hline & TWIST1 & & & 132 & 109 & -2.51 & 0.010 \\
\hline & ZEB2 & & & 138 & 106 & -3.49 & $<0.001$ \\
\hline & ZEB1 & & & 134 & 108 & -2.825 & 0.005 \\
\hline & $S L U G$ & & & 129 & 111 & -2.048 & 0.040 \\
\hline & SNAIL & & & 113 & 121 & -0.94 & 0.340 \\
\hline & $N F K B 1$ & & & 115 & 119 & -0.47 & 0.630 \\
\hline & $L L G L 2$ & & & 89 & 135 & -5.00 & $<0.001$ \\
\hline & GSK3B & & & 108 & 124 & -1.71 & 0.080 \\
\hline & CRUMBS & & & 91 & 135 & -4.80 & $<0.001$ \\
\hline
\end{tabular}

*Significant $p$-values are in bold

*Dichotomization of mRNA level was based on median of the total expression of each gene
Table 3 Correlation between mRNA level of the genes associated with E-cadherin expression in triple negative high-grade invasive ductal carcinoma in the METABRIC cohort

\section{E-cadherin loss and expression of genes differentially expressed between invasive lobular carcinoma and invasive ductal carcinoma within the triple-negative breast cancer cohort}

There was no significant association between reduced/ loss of E-cadherin expression in the high-grade triple-negative ductal cancer and those genes differentially expressed between invasive lobular and ductal carcinoma (Cathepsin B, TPII, SPRY1, SCYA14, TFAP2B, thrombospondin 4, Osteopontin, HLA-G, CHCl) [27] (Table 5 and Supplementary Figure 4B).

\section{Genomic study and pathway analysis}

Next-generation sequencing identified 2143 differentially expressed genes (Benjamin-Hochberg; $p<0.05$, differentially expressed by $>$ two-fold, false discovery rate $<0.05$ ). Triple-negative invasive ductal carcinoma with reduced/loss E-cadherin expression $(n=46)$ showed 849 significantly overexpressed and 1294 downregulated genes. It is noteworthy that dysregulation of genes regulating Wnt signaling pathway, the top predicted master regulator of E-cadherin expression, based on $p$-value, whose activity could explain protein expression differences were FZD2, GNG5, HLTF, 
Table 4A Correlation between level of proteins known to control E-cadherin expression using triple-negative invasive breast carcinoma cohort $(n=$ 106)

\begin{tabular}{|c|c|c|c|c|c|c|c|c|}
\hline \multirow[t]{2}{*}{ Identifier } & & \multirow[t]{2}{*}{$\begin{array}{l}\text { Protein of } \\
\text { interest }\end{array}$} & \multicolumn{2}{|c|}{$\begin{array}{l}\text { E-cadherin } \\
\text { expression } \\
\text { frequency }\end{array}$} & \multicolumn{2}{|c|}{$\begin{array}{l}\text { Mean } \\
\text { RANK }\end{array}$} & \multirow[t]{2}{*}{ Z- score } & \multirow[t]{2}{*}{$p$-Value } \\
\hline & & & Low $(\%)$ & High $(\%)$ & Low & High & & \\
\hline \multirow[t]{11}{*}{ Protein level } & Transcription factor & TGFBeta1 & $27(47)$ & $30(53)$ & 27 & 31 & -0.87 & 0.380 \\
\hline & & TWIST2 & $26(48)$ & $28(52)$ & 27 & 31 & -0.23 & 0.810 \\
\hline & & ZEB & $25(49)$ & $26(51)$ & 27 & 25 & -0.79 & 0.430 \\
\hline & DNA repair family & BRCA1 & $33(44)$ & $41(56)$ & 37 & 38 & -0.26 & 0.790 \\
\hline & & BRCA2 & $26(45)$ & $31(55)$ & 26 & 32 & -1.90 & 0.060 \\
\hline & & GammaH2ax & $27(43)$ & $35(57)$ & 28 & 34 & -1.47 & 0.140 \\
\hline & & RAD51 & $23(51)$ & $22(49)$ & 21 & 24 & -0.85 & 0.390 \\
\hline & $\begin{array}{l}\text { Proliferation and } \\
\text { other markers family }\end{array}$ & Ki67 & $35(47)$ & $40(53)$ & 42 & 34 & -1.61 & 0.100 \\
\hline & & PTEN & $21(47)$ & $19(53)$ & 22 & 19 & -0.96 & 0.390 \\
\hline & & ATM & $28(49)$ & $29(51)$ & 29 & 29 & -0.18 & 0.850 \\
\hline & & TP53 & $39(41)$ & $45(59)$ & 43 & 42 & -0.32 & 0.750 \\
\hline
\end{tabular}

\begin{tabular}{|c|c|c|c|c|c|c|c|c|}
\hline \multirow[t]{2}{*}{ Identifier } & & \multirow[t]{2}{*}{$\begin{array}{l}\text { Gene of } \\
\text { interest }\end{array}$} & \multicolumn{2}{|c|}{$\begin{array}{l}\text { E-cadherin expression } \\
\text { frequency }\end{array}$} & \multicolumn{2}{|c|}{$\begin{array}{l}\text { Mean } \\
\text { RANK }\end{array}$} & \multirow[t]{2}{*}{$Z$ - score } & \multirow[t]{2}{*}{$p$-Value } \\
\hline & & & Low (\%) & High (\%) & Low & High & & \\
\hline \multirow[t]{11}{*}{$\begin{array}{l}\text { mRNA } \\
\text { level }\end{array}$} & $\begin{array}{l}\text { Transcription factor } \\
\text { family }\end{array}$ & $T G F B 1$ & \multirow{11}{*}{$46(43)$} & \multirow{11}{*}{$60(57)$} & 56 & 51 & -0.88 & 0.370 \\
\hline & & TWIST2 & & & 56 & 51 & -1.28 & 0.200 \\
\hline & & TWIST1 & & & 53 & 53 & -0.01 & 0.990 \\
\hline & & ZEB2 & & & 56 & 52 & -0.74 & 0.450 \\
\hline & & ZEB1 & & & 57 & 51 & -0.94 & 0.340 \\
\hline & & $S L U G$ & & & 51 & 55 & -0.69 & 0.480 \\
\hline & & SNAIL & & & 55 & 53 & -0.43 & 0.660 \\
\hline & & $N F K B 1$ & & & 57 & 51 & -0.95 & 0.340 \\
\hline & & $L L G L 2$ & & & 58 & 50 & -1.33 & 0.180 \\
\hline & & GSK3B & & & 54 & 53 & -0.29 & 0.760 \\
\hline & & CRUMBS & & & 53 & 54 & -0.07 & 0.930 \\
\hline
\end{tabular}

WNT2, and CER1; PIK3-AKT signaling pathway top predicted master regulator controlling E-cadherin expression were FGFR2, GNF5, GNGT1, IFNA17, and IGF1 (Table 6). Importantly, key genes differentially expressed between invasive lobular carcinoma and invasive ductal tumors [27] did not show association with E-cadherin reduced/loss of expression in the invasive triple-negative ductal carcinoma (Table 5).

\section{Discussion}

Reduced/loss of E-cadherin expression is recognized as part of the main molecular events driving loss of cell-cell adhesion and thus facilitating cancer invasion and metastasis [28]. Some authors have suggested that E-cadherin can serve as a phenotypic marker to distinguish between invasive lobular carcinoma and non-invasive lobular tumors [27]. Mechanisms seeding reduced/loss of E-cadherin expression comprise $\mathrm{CDHl}$ gene mutation [10], truncating mutation [29], promoter hypermethylation [30], and transcriptional inactivation [31]. Reduced/loss of E-cadherin expression is observed in $84 \%$ of invasive lobular carcinomas [9]. Several studies have shown that $38 \%$ of highgrade invasive ductal tumors show reduced/ loss of Ecadherin expression and this phenomenon has been linked to aggressive tumor behavior. Interestingly, $\mathrm{CDH} 1$ gene mutations were not identified in this subgroup [11, 19, 32]. 
Table 5 Genes differentially expressed between lobular vs. ductal breast carcinomas in triple-negative breast cancer cohort $(n=106)$

\begin{tabular}{|c|c|c|c|c|c|c|c|}
\hline \multirow[t]{2}{*}{ Identifier } & \multirow[t]{2}{*}{ Gene of interest } & \multicolumn{2}{|c|}{$\begin{array}{l}\text { E-cadherin expression } \\
\text { frequency }\end{array}$} & \multicolumn{2}{|c|}{ Mean RANK } & \multirow[t]{2}{*}{$Z$ - score } & \multirow[t]{2}{*}{$p$-Value } \\
\hline & & Low $(\%)$ & High (\%) & Low & High & & \\
\hline \multirow[t]{9}{*}{ mRNA Level } & Cathepsin B & \multirow{9}{*}{$46(43)$} & \multirow{9}{*}{$60(57)$} & 51 & 55 & -0.57 & 0.560 \\
\hline & TPII & & & 56 & 51 & -0.87 & 0.380 \\
\hline & $S P R Y 1$ & & & 54 & 53 & -0.39 & 0.690 \\
\hline & SCYA14 & & & 54 & 53 & -0.20 & 0.830 \\
\hline & $T F A P 2 B$ & & & 52 & 55 & -0.46 & 0.640 \\
\hline & Thrombospondin 4 & & & 53 & 54 & -0.09 & 0.920 \\
\hline & Osteopontin & & & 54 & 53 & -0.26 & 0.790 \\
\hline & $H L A-G$ & & & 52 & 55 & -0.51 & 0.600 \\
\hline & $\mathrm{CHCl}$ & & & 53 & 54 & -0.13 & 0.890 \\
\hline
\end{tabular}

Table 6 Pathway analysis results using Webgestalt to identify differentially regulated canonical pathways in the triplenegative breast cancer cohort

\begin{tabular}{llllll}
\hline Master regulator & $\begin{array}{l}\text { Gene } \\
\text { symbol }\end{array}$ & Gene name & Score & $\begin{array}{l}\text { FDR score } \\
\text { from GSEA }\end{array}$ & $\begin{array}{l}p \text {-Value from } \\
\text { GSEA }\end{array}$ \\
\hline $\begin{array}{l}\text { PI3K-Akt signaling } \\
\text { pathway }\end{array}$ & FGFR2 & $\begin{array}{l}\text { Fibroblast growth factor } \\
\text { receptor 2 }\end{array}$ & 0.04 & 0.0069 & $<\mathbf{0 . 0 0 1}$ \\
& GNG5 & G protein subunit gamma 5 & 0.03 & & \\
& GNGT1 & G protein subunit gamma & 0.02 & & \\
& transducin1 & 0.03 & & \\
& IFNA17 & Interferon alpha 17 & 0.03 & \\
& IGF1 & Insulin like growth factor & 0.01 & 0.0024 & \\
Wnt signaling pathway & FZD2 & Frizzled class receptor 2 & 0.03 & \\
& GNG5 & G protein subunit gamma 5 & & \\
& HLTF & Helicase like transcription factor & 0.01 & & \\
& $W N T 2$ & Wnt family member 2 & 0.03 & & \\
& CER1 & Cerberus 1, DAN family BMP & 0.01 & & \\
& antagonist & & & \\
\hline
\end{tabular}

Significant $P$-values are in bold
One of the recognized mechanisms leading to reduced/ loss of E-cadherin expression is loss of heterozygosity at chromosome 16q22.1, where the CHD1 gene is located [33]. Studies investigating the mechanism underlying reduced/loss of E-cadherin protein expression in invasive lobular carcinoma cases uncovered loss of wild-type allele due to loss of heterozygosity at $16 \mathrm{q} 22.1$ occurring in $>70 \%$ of cases [7,8]. Furthermore, $\mathrm{CDH1}$ gene mutation and promoter hypermethylation were observed in $20 \%$ and $56 \%$ of invasive lobular carcinomas, respectively [7]. Interestingly, co-occurrence of these mechanisms rarely occurs in invasive lobular tumors [34]. Remarkably, mutational inactivation of $\mathrm{CDH} 1$ gene mostly coexists with loss of the wild-type allele in invasive lobular carcinoma [35]. As reduced/loss of E-cadherin expression in invasive lobular tumors is predominantly caused by loss of heterozygosity, it has been suggested that copy number loss of the $\mathrm{CDH} 1$ gene can be used to discriminate between invasive ductal carcinoma and invasive lobular tumors when it is difficult to differentiate them based on histological evaluation [36]. Our investigation revealed that copy number loss occurred in only $21 \%$ of invasive ductal carcinomas displaying reduced/ loss of E-cadherin expression. Therefore, other mechanisms must underlie the downregulation of E-cadherin in the majority of cases. Other mechanisms of E-cadherin reduced/ loss of expression without copy number loss include DNA hypermethylation, a mechanism that may induce the $\mathrm{CDH} 1$ reduced/loss of mRNA expression detected in $60 \%$ of metastatic invasive ductal carcinoma [37].

Loss of $\mathrm{CDH1}$ gene at 16q22.1 in invasive lobular carcinoma is one of the main genetic events and is observed early in the process of carcinogenesis in lobular carcinomas. We hypothesized that reduced/loss of E-cadherin expression in a subset of invasive ductal tumors might be the result of genomic instability and occurs as a late event during the process of cancer progression. Our results demonstrate that loss of $\mathrm{CDH} 1$ copy number is associated with copy number aberrations of multiple well-established breast cancer tumor 
suppressor genes located at different chromosomes; copy number loss of ATM (11q22.3), PTEN (10q23.31), RBI (13q14.2), TP53 (17p13.1), BRCA1 (17q21.31), and BRCA2 (13q13.1) tumor suppressor genes. Moreover, DNA damage response pathways, which are crucial for detecting DNA lesions and arresting the cell cycle until the DNA is repaired or inducing cell death if cells sustain irreparable DNA damage [38], have key roles in preventing genetic instability and tumorigenesis [39]. Investigation of correlations between reduced/loss of E-cadherin expression and expression of biomarkers related to DNA damage response pathways in breast cancer revealed negative correlation between reduced/ loss of E-cadherin protein expression and GammaH2AX and PTEN expression, suggesting that reduced/loss of E-cadherin expression is associated with impaired DNA damage response and, likely, genomic instability. Taken together, these results support our hypothesis that reduced/loss of E-cadherin expression in invasive ductal carcinomas is associated with genomic instability.

Reduced/loss of E-cadherin expression can also be caused by overexpression of its associated transcription factors $[40-42,26]$. Our results showed a negative correlation between reduced/loss of $C D H 1$ mRNA expression and the mRNA expression of transcription factors known to suppress E-cadherin expression and cause disruption of cell-cell adhesion [43, 44]; in fact, $76 \%$ of cases harboring reduced/loss of $C D H 1$ mRNA show upregulation of one or more of these transcriptional repressors.

Remarkably, other key factors in epithelial-mesenchymal transition such as TGFBeta1, SNAIL, and SLUG did not show any correlation with E-cadherin reduced/loss of mRNA expression. These observations suggest that reduced/ loss of E-cadherin expression is not merely a surrogate for epithelial-mesenchymal transition but represents a readout of other pathways controlling E-cadherin expression at membranes level.

Of note, reduced/loss of E-cadherin protein expression occurs in up to $50 \%$ of triple-negative invasive ductal carcinoma, which may contribute to increased lymph node metastasis, and poor patient outcomes [45]. We observed a negative correlation between reduced/loss of $\mathrm{CDH} 1 \mathrm{mRNA}$ expression and the mRNA expression of multiple transcription factors known to suppress E-cadherin expression in our triple-negative breast cancer cohort. On the contrary, when we investigated the same genotype within the cohort tested by next-generation sequencing, none of these transcription factors showed statistically significant associations with E-cadherin expression. It is possible that different molecular mechanisms regulate E-cadherin expression, although we cannot exclude the possibility that our cohort is small to such associations.

More importantly, genes differentially expressed between invasive ductal and invasive lobular breast tumors as identified by Waldman et al. [27] and could represent the effect of E-cadherin loss in lobular carcinoma compared with ductal tumors showed no statistically significant difference, when tested on mRNA level, in breast cancer cases, showing reduced/loss of E-cadherin expression compared with these tumor with normal expression. This may indicate not only that more complex molecular mechanisms are responsible for E-cadherin reduced/loss of protein expression in these cases but also E-cadherin loss in ductal carcinoma does not produce the same effects in lobular tumors. This may also be supported by the lack of morphological features and metastatic behavior characteristic of lobular carcinomas in ductal tumors lacking E-cadherin expression.

In this study, differential gene expression using nextgeneration sequencing investigating differences between cases with reduced/loss of E-cadherin expression and cases with normal/high expression showed dysregulation of genes regulating PIK3-AKT signaling pathway. Our analysis exposed a negative correlation between the genes regulating this pathway and reduced/loss of E-cadherin protein expression, suggesting that overexpression of those indicators may promote signaling via the PIK3-AKT pathway and thus negatively regulate E-cadherin expression. Receptors such as insulin-like growth factor receptor 1 can induce the activity of Akt pathway [46]. Our results are in agreement with reports indicating activation of PIK3-AKT represses E-cadherin expression and stimulates cell migration [47]. Nonetheless, dysregulation of genes regulating Wnt signaling pathway was also present in our results. Mutation or deregulation of gene expression of the canonical Wnt pathway is implicated in cancer [48-50].

Our study limitation relates to comparing gene expression data obtained from microarrays, as used in the METABRIC cohort comprising different molecular subtypes of invasive ductal tumors, and the RNA-Seq dataset available for our triple-negative breast cancers only. We have chosen triple-negative breast cancer to study E-cadherin protein expression in invasive ductal carcinoma cases, as up to $50 \%$ of this molecular subtype show reduced/loss of E-cadherin protein expression $[45,51]$. On the contrary, studies have shown that reduced/loss of E-cadherin expression occurs in $23 \%$ and $27 \%$ of luminal and HER2 enriched subtypes, respectively [51]. RNA-Seq approaches cover multiple aspects of the transcriptome without any a priori knowledge, allowing to identify novel transcripts, splice junctions, and noncoding RNAs [52]. We acknowledge that comparison between these two different approaches may or may not provide the same results due to intrinsic differences in assay design [53]. For instance, next-generation sequencing may have different lower limits of detection or may encompass different genomic regions [52]. More importantly, 
invasive ductal carcinoma cases used in the METABRIC cohort comprise different molecular subtypes, whereas the RNA-Seq data were acquired for a triple-negative breast cancer cohort, which also may have a role in our study. Therefore, further validation of our findings is warranted.

\section{Conclusion}

Reduced/loss E-cadherin expression in invasive ductal carcinoma is a complex biological phenomenon, which, according to the findings of this study, appears to be a part of the genomic instability process occurring late in the process of carcinogenesis rather than an initial neoplastic event and results in different effects to those produced in invasive lobular carcinomas. Using the high-throughput next-generation sequencing, we have unraveled potential novel regulators controlling different signaling pathways that regulate E-cadherin protein expression in invasive ductal carcinoma. These regulators warrant further investigation and validation using different platforms.

Acknowledgements We thank the Nottingham Health Science Biobank and Breast Cancer Now Tissue Bank for the provision of tissue samples.

\section{Compliance with ethical standards}

Conflict of interest The authors declare that they have no conflict of interest.

Publisher's note: Springer Nature remains neutral with regard to jurisdictional claims in published maps and institutional affiliations.

\section{References}

1. Bray F, Ferlay J, Soerjomataram I, et al. Global cancer statistics 2018: GLOBOCAN estimates of incidence and mortality worldwide for 36 cancers in 185 countries. CA Cancer J Clin. 2018; 68:394-424.

2. Hart IR, Saini A. Biology of tumour metastasis. Lancet (Lond, Engl). 1992;339:1453-7.

3. Berx G, Staes K, van Hengel J, et al. Cloning and characterization of the human invasion suppressor gene E-cadherin (CDH1). Genomics. 1995;26:281-9.

4. Paredes J, Figueiredo J, Albergaria A, et al. Epithelial E- and P-cadherins: role and clinical significance in cancer. Biochim Biophys Acta Rev Cancer 2012;1826:297-311.

5. Behrens J, Mareel MM, Van Roy FM, et al. Dissecting tumor cell invasion: epithelial cells acquire invasive properties after the loss of uvomorulin-mediated cell-cell adhesion. J Cell Biol. 1989;108:2435-47.

6. Frixen UH, Behrens J, Sachs M, et al. E-cadherin-mediated cellcell adhesion prevents invasiveness of human carcinoma cells. J Cell Biol. 1991;113:173-85.

7. Sarrió D, Moreno-Bueno G, Hardisson D, et al. Epigenetic and genetic alterations of APC and $\mathrm{CDH} 1$ genes in lobular breast cancer: relationships with abnormal E-cadherin and catenin expression and microsatellite instability. Int J Cancer. 2003;106:208-15.
8. Huiping C, Sigurgeirsdottir JR, Jonasson JG, et al. Chromosome alterations and E-cadherin gene mutations in human lobular breast cancer. Br J Cancer. 1999;81:1103-10.

9. de Leeuw WJF, Berx G, Vos CBJ, et al. Simultaneous loss of E-cadherin and catenins in invasive lobular breast cancer and lobular carcinomain situ. J Pathol. 1997;183:404-11.

10. Sgeirsson KSA, Joâ Nasson JG, Tryggvadoâ Ttir L, et al. Altered expression of E-cadherin in breast cancer: patterns, mechanisms and clinical signi ${ }^{\circledR}$ cance [cited 12 July 2018]. Available from: https://skemman.is/bitstream/1946/21610/1/ Asgeirsson2000.pdf.

11. Acs G, Lawton TJ, Rebbeck TR, et al. Differential expression of Ecadherin in lobular and ductal neoplasms of the breast and its biologic and diagnostic implications. Am J Clin Pathol. 2001;115:85-98.

12. Rakha EA, Teoh TK, Lee AHS, et al. Further evidence that E-cadherin is not a tumour suppressor gene in invasive ductal carcinoma of the breast: an immunohistochemical study. Histopathology. 2013;62:695-701.

13. Jeschke U, Mylonas I, Kuhn C, et al. Expression of E-cadherin in human ductal breast cancer carcinoma in situ, invasive carcinomas, their lymph node metastases, their distant metastases, carcinomas with recurrence and in recurrence. Anticancer Res. 2007;27:1969-74.

14. da Silva BB, dos Santos AR, Pires CG, et al. E-cadherin expression in estrogen receptor-positive and negative breast carcinomas of postmenopausal women. Eur J Gynaecol Oncol. 2010;31:90-93.

15. Siitonen SM, Kononen JT, Helin HJ, et al. Reduced E-cadherin expression is associated with invasiveness and unfavorable prognosis in breast cancer. Am J Clin Pathol. 1996;105:394-402.

16. Mahler-Araujo B, Savage K, Parry S, et al. Reduction of E-cadherin expression is associated with non-lobular breast carcinomas of basal-like and triple negative phenotype. J Clin Pathol. 2008;61:615-20.

17. Lipponen P, Saarelainen E, Ji H, et al. Expression of E-cadherin (E-CD) as related to other prognostic factors and survival in breast cancer. J Pathol. 1994;174:101-9.

18. McCart Reed AE, Kutasovic JR, Lakhani SR, et al. Invasive lobular carcinoma of the breast: morphology, biomarkers and' omics. Breast Cancer Res. 2015;17:12.

19. Gillett CE, Miles DW, Ryder K, et al. Retention of the expression of E-cadherin and catenins is associated with shorter survival in grade III ductal carcinoma of the breast. J Pathol. 2001;193: 433-41.

20. Rakha EA, Abd El Rehim D, Pinder SE, et al. E-cadherin expression in invasive non-lobular carcinoma of the breast and its prognostic significance. Histopathology. 2005;46:685-93.

21. Curtis C, Shah SP, Chin S-F, et al. The genomic and transcriptomic architecture of 2,000 breast tumours reveals novel subgroups. Nature. 2012;486:346-52.

22. Šidák Z. Rectangular confidence regions for the means of multivariate normal distributions. J Am Stat Assoc. 1967;62:626-33.

23. McCarty KS, Miller LS, Cox EB, et al. Estrogen receptor analyses. Correlation of biochemical and immunohistochemical methods using monoclonal antireceptor antibodies. Arch Pathol Lab Med. 1985;109:716-21.

24. Lohse M, Bolger AM, Nagel A, et al. RobiNA: a user-friendly, integrated software solution for RNA-Seq-based transcriptomics. Nucleic Acids Res. 2012;40:W622-W627.

25. Wang J, Vasaikar S, Shi Z, et al. WebGestalt 2017: a more comprehensive, powerful, flexible and interactive gene set enrichment analysis toolkit. Nucleic Acids Res. 2017;45: W130-W137.

26. Baranwal S, Alahari SK. Molecular mechanisms controlling E-cadherin expression in breast cancer. Biochem Biophys Res Commun. 2009;384:6-11. 
27. Korkola JE, DeVries S, Fridlyand J, et al. Differentiation of lobular versus ductal breast carcinomas by expression microarray analysis. Cancer Res. 2003;63:7167-75.

28. Debies MT, Welch DR. Genetic basis of human breast cancer metastasis. J Mammary Gland Biol Neoplasia. 2001;6:441-51.

29. Strathdee G. Epigenetic versus genetic alterations in the inactivation of E-cadherin. Semin Cancer Biol. 2002;12:373-9.

30. Graff JR, Herman JG, Lapidus RG, et al. E-cadherin expression is silenced by DNA hypermethylation in human breast and prostate carcinomas. Cancer Res. 1995;55:5195-9.

31. Batlle E, Sancho E, Francí C, et al. The transcription factor Snail is a repressor of E-cadherin gene expression in epithelial tumour cells. Nat Cell Biol. 2000;2:84-89.

32. Oka H, Shiozaki H, Kobayashi K, et al. Expression of E-cadherin cell adhesion molecules in human breast cancer tissues and its relationship to metastasis. Cancer Res. 1993;53:1696-701.

33. Cleton-Jansen AM, Callen DF, Seshadri R, et al. Loss of heterozygosity mapping at chromosome arm 16q in 712 breast tumors reveals factors that influence delineation of candidate regions. Cancer Res. 2001;61:1171-7.

34. Cheng C-W, Wu P-E, Yu J-C, et al. Mechanisms of inactivation of E-cadherin in breast carcinoma: modification of the two-hit hypothesis of tumor suppressor gene. Oncogene. 2001;20:3814-23.

35. Berx G, Cleton-Jansen AM, Strumane K, et al. E-cadherin is inactivated in a majority of invasive human lobular breast cancers by truncation mutations throughout its extracellular domain. Oncogene. 1996;13:1919-25.

36. Li J, Wang K, Li S, et al. DNA copy number aberrations in breast cancer by array comparative genomic hybridization. Genom Proteom Bioinformatics. 2009;7:13-24.

37. Caldeira JRF, Prando ÉC, Quevedo FC, et al. CDH1promoter hypermethylation and E-cadherin protein expression in infiltrating breast cancer. BMC Cancer. 2006;6:48.

38. Sengodan SK, K.H. S, Nadhan R, et al. Regulation of epithelial to mesenchymal transition by BRCA1 in breast cancer. Crit Rev Oncol Hematol. 2018;123:74-82.

39. Yasmeen A, Liu W, Dekhil H, et al. BRCA1 mutations contribute to cell motility and invasion by affecting its main regulators. Cell Cycle. 2008;7:3781-3

40. Becker KF, Atkinson MJ, Reich U, et al. E-cadherin gene mutations provide clues to diffuse type gastric carcinomas. Cancer Res. 1994;54:3845-52.
41. Berx G, Cleton-Jansen AM, Nollet F, et al. E-cadherin is a tumour/invasion suppressor gene mutated in human lobular breast cancers. EMBO J. 1995;14:6107-15.

42. Cleton-Jansen A-M. E-cadherin and loss of heterozygosity at chromosome 16 in breast carcinogenesis: different genetic pathways in ductal and lobular breast cancer? Breast Cancer Res. 2002;4:5-8

43. Spaderna S, Schmalhofer O, Wahlbuhl M, et al. The transcriptional repressor ZEB1 promotes metastasis and loss of cell polarity in cancer. Cancer Res. 2008;68:537-44.

44. Aigner K, Dampier B, Descovich L, et al. The transcription factor ZEB1 ( $\delta E F 1)$ promotes tumour cell dedifferentiation by repressing master regulators of epithelial polarity. Oncogene. 2007;26:6979-88.

45. Kashiwagi S, Yashiro M, Takashima T, et al. Significance of E-cadherin expression in triple-negative breast cancer. Br J Cancer. 2010;103:249-55.

46. Foulstone E, Prince $\mathrm{S}$, Zaccheo $\mathrm{O}$, et al. Insulin-like growth factor ligands, receptors, and binding proteins in cancer. J Pathol. 2005;205:145-53.

47. Irie HY, Pearline RV, Grueneberg D, et al. Distinct roles of Akt1 and Akt2 in regulating cell migration and epithelial-mesenchymal transition. J Cell Biol. 2005;171:1023-34.

48. Bienz M, Clevers H. Linking colorectal cancer to Wnt signaling. Cell. 2000;103:311-20.

49. Prasad CP, Gupta SD, Rath G, et al. Wnt signaling pathway in invasive ductal carcinoma of the breast: relationship between beta-catenin, dishevelled and cyclin D1 expression. Oncology. 2007;73:112-7.

50. Prasad CP, Mirza S, Sharma G, et al. Epigenetic alterations of CDH1 and APC genes: Relationship with activation of Wnt/ $\beta$-catenin pathway in invasive ductal carcinoma of breast. Life Sci. 2008;83:318-25.

51. Liu J-B, Feng C-Y, Deng $M$, et al. E-cadherin expression phenotypes associated with molecular subtypes in invasive non-lobular breast cancer: evidence from a retrospective study and meta-analysis. World J Surg Oncol. 2017;15:139.

52. Hurd PJ, Nelson CJ. Advantages of next-generation sequencing versus the microarray in epigenetic research. Brief Funct Genom Proteom. 2009;8:174-83.

53. Wolff A, Bayerlová M, Gaedcke J, et al. A comparative study of RNA-Seq and microarray data analysis on the two examples of rectal-cancer patients and Burkitt Lymphoma cells. PLoS ONE. 2018;13:e197162.

\section{Affiliations}

\section{Mansour Alsaleem ${ }^{1,2} \cdot$ Michael S. Toss $\mathbb{D}^{1} \cdot$ Chitra Joseph $^{1}{ }^{1} \cdot$ Mohammed Aleskandarany $^{1,3} \cdot$ Sasagu Kurozumi ${ }^{1}$. Ibrahim Alshankyty ${ }^{4}$ Angela Ogden ${ }^{5} \cdot$ Padmashree C. G. Rida $^{5} \cdot$ lan O. Ellis ${ }^{1} \cdot$ Ritu Aneja $^{5} \cdot$ Andrew R. Green $\mathbb{D}^{1}$ • Nigel P. Mongan $\mathbb{1}^{6,7} \cdot$ Emad A. Rakha ${ }^{1,3}$}

1 Nottingham Breast Cancer Research Centre, Division of Cancer and Stem Cells, School of Medicine, University of Nottingham, Nottingham, UK

2 Faculty of Applied Medical Sciences, Onizah Community College, Qassim University, Qassim, Saudi Arabia

3 Faculty of Medicine, Menoufyia University, Shebin El Kom, Egypt

4 Faculty of Applied Medical Sciences, King Abdulaziz University,
Jeddah, Saudi Arabia

5 Department of Biology, Georgia State University, Atlanta, GA, USA

6 Cancer Biology and Translational Research, Faculty of Medicine and Health Sciences, University of Nottingham, Nottingham, UK

7 Department of Pharmacology, Weill Cornell Medicine, New York, USA 